\title{
Synthesis and Biological Evaluation of N-Heterocyclic Substituted Fluoro-Benzothiazole Sulphonamido Analogs as a Potential Therapeutic Agents
}

\author{
Sri Ranga.T* ${ }^{*}$ Neelesh Chaubey
}

\section{College of Pharmacy, Sri Satya Sai University of Technology and Medical sciences, Sehore} (M.P)

Article Info: Received 15 October 2021; Accepted 10 December. 2021

DOI: https://doi.org/10.32553/jbpr.v11i1.893

Address for Correspondence: Sri Ranga.T*

Conflict of interest statement: No conflict of interest

\section{Abstract:}

The present investigation is aimed to synthesize fluorobenzothiazole comprising sulphonamido pyrazole analogs starting from fluoro-chloroaniline to get 2-amino-6-fluoro-7-chloro $(1,3)$ benzothiazole (I), this was treated with anilino-s-methyl ethylene cyanoacetamide in the presence of ethanol to get desired molecules. The synthesized targeted molecules are characterized, docked and screened for their invitro antidiabetic properties.

Keywords: Fluorobenzothiazole, Docking, antidiabetic

\section{Introduction}

Fluorobenzothiazole ${ }^{1}$ comprising sulfonamide pyrazole derivatives have been synthesized and evaluated for their various activities.

The sulfonamide ${ }^{2-5}$ drugs were the first effective chemotherapeutic agents to be employed systemically for the prevention and cure of bacterial infection in human beings. The introduction of trimethaprim and sulphamethoxazole has resulted in increased use of sulfonamide for the treatment of specific microbial infection. Benzothiazoles with sulphonyl group and pyrazole etc were reported to posses various pharmacological activity of clinical importance.

The chemistry and pharmacology of pyrazole ${ }^{6-10}$ have been of great interest because pyrazole derivatives possess various biological activities. Therefore in present work we have prepared sulphonamido-pyrazole analogs incorporate with substituted benzothiazole.
Molecular docking studies ${ }^{11-13}$ are used to determine the interaction of two molecules and to find the best orientation of ligand which would form a complex with overall minimum energy. The small molecule, known as ligand usually fits within protein's cavity which is predicted by the search algorithm. These protein cavities become active when they come in contact with any external compounds and are thus called as active sites.

Docking is frequently used to predict the binding orientation of small molecule drug candidates to their protein targets in order to predict the affinity and activity of the small molecule. Hence docking plays an important role in the rational drug design.

\section{Materials and methods:}

Melting point was determined by open capillary tube method and are uncorrected. 
T.L.C was run on silica gel G plates using nbutyl alcohol, ethyl acetate and carbontertachloride $(1: 2: 1)$ as developing solvent for the purity of the compounds. I.R. Spectra were recorded on Shimadzu FTIR Spectrophotometer by using $\mathrm{KBr}$ pellet technique.

Experimental:

Synthesis of 6-fluoro-7-substituted-2-[(3'amino-4'-carboxamido-5'-s-methyl-

pyrazolidin-1'-yl)-p-benzene sulphonamido] $(1,3)$ - benzothiazole.

A mixture of 6-fluoro-7-substituted-2-( $p$ hydrazino benzene sulphonamido)-(1,3)benzothiazole and anilino-s-methyl ethylene cyanoacetamide were refluxed in ethanol for 2 hrs. and later excess of ethanol was distilled off and poured onto crushed ice. The obtained was filtered and was recrystallised from ethanol.

6-fluoro-7-substituted-2-(3'-amino-4'carboxamido-5'-anilino-pyrazolidin-1'-yl- phenyl- $p$-sulphonamido) (1,3)- benzothiazole was taken with 0.015 mol solution of aldehydes (p-dimethylaminobenzaldehyde) in round bottom flask, then added $20 \mathrm{ml}$ of ethanol and 3-4 drops of $\mathrm{HCl}$ and refluxed for $2-3$ hours, then the mixture in concentrated to remove ethanol. The remaining solution is cooled and poured in to crushed ice in small portions to get targeted molecules.

\section{Results and Discussion:}

\section{1) INSILICO ANTIDIABETIC activity for SRT Compounds:}

The synthesized compounds of SRT 1-12 were submitted to in-silico evaluation by using molecular docking approach. Insilico screening for antidiabetic activity was done by using Autodock. The selected target is Insulin Receptor Tyrosine Kinase enzyme, with PDB ID-2B4S, consisting of resolution $\mathbf{2 . 3 0}$ Angstroms.

Table 1: DOCKING RESULTS for SRT 1-12 Compounds.

\begin{tabular}{|l|l|l|}
\hline S1 No & Compound name & Docking score \\
\hline 1. & SRT1 & -7.6 \\
\hline 2. & SRT2 & -7.3 \\
\hline 3. & SRT3 & -7.9 \\
\hline 4. & SRT4 & -8.2 \\
\hline $\mathbf{5 .}$ & SRT5 & $\mathbf{- 8 . 6}$ \\
\hline 6. & SRT6 & -8.4 \\
\hline 7. & SRT7 & -7.8 \\
\hline 8. & SRT & -8.3 \\
\hline 9. & SRT9 & -8.0 \\
\hline 10. & SRT10 & -7.6 \\
\hline 11. & SRT11 & -8.3 \\
\hline 12. & SRT12 & -6.9 \\
\hline 13. & METFORMINE & -5.6 \\
\hline 14. & GLIMEPIRIDE & -7.4 \\
\hline
\end{tabular}

Order of showing potent antidiabetic activity:

SRT5 $>$ SRT6 $>$ SRT11 $=$ SRT $>$ SRT4 $>$ SRT9 $>$ SRT3 $>$ SRT7 $>$ SRT1 $=$ SRT10 $>$ SRT2 $>$ SRT $>12>$ GLIMIPRIDE $>$ METFORMIN. 
Glimipride and Metfromin are the standard Drugs.Among the all synthesized SRT compounds SRT5 exhibited more potent antidiabetic activity than other compounds. The SRT5 exhibited binding energy with -8.6Kcal.
Remaining all the synthesized compounds also exhibit moderate activity when compared with Standard drug Glimipride and Metfromin. The Standard drugs Glimipride and Metfromin Binding energy is -7.4 and -5.6

\section{Docking Results:}

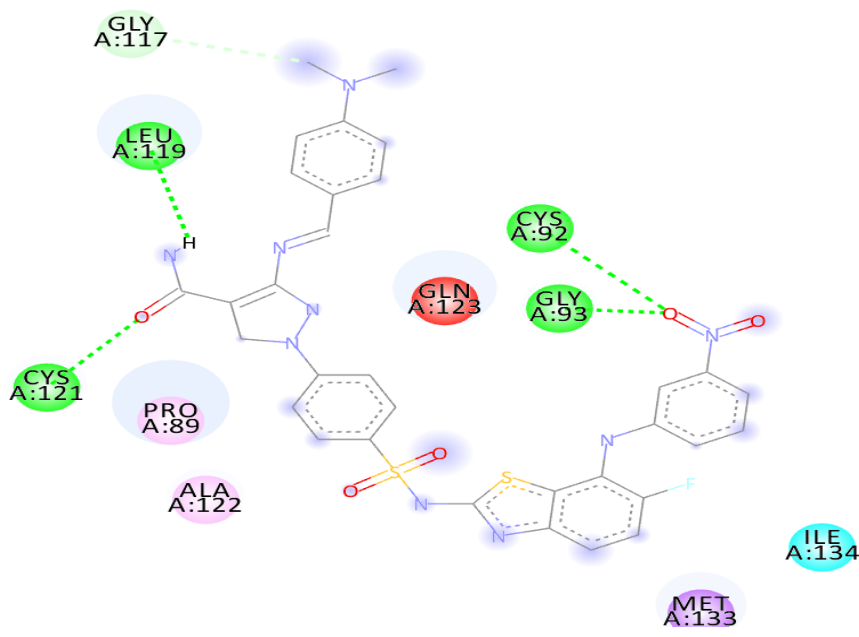

2D Structure of SRT-5:

By analyzing of the 2D results of SRT-5 it involved mainly Hydrogen Bonding . The ligand interacts mainly Hydrogen bonding with aminoacid Leucine A119, CYSA121, 92 and Glycine A93. Remaining all SRT Compounds involved mainly Hydrogen Bonding and Hydrophobic interactions with Ligand

\section{In-vitro Antidiabetic Activity: ${ }^{14-17}$}

\section{Procedure:}

3T3-L1 adipocytes, were seeded at a density of $\sim 1500$ cells per well in a 96-well plate, differentiated and maintained for another 10 days prior to use. To assay glucose uptake, adipocytes were starved in $100 \mu \mathrm{l}$ serum free adipocyte medium overnight (to enhance glucose uptake) then washed with PBS, followed by a incubation (40 min) in an glucose free medium (100 $\mu$ l Krebs-Ringer-PhosphateHEPES (KRPH) buffer with $2 \%$ BSA) then stimulated either with insulin (PGZ) $(10 \mu \mathrm{M})$, compounds $(10 \mu \mathrm{g} / \mathrm{ml})$ or PBS. $10 \mu 1$ of $10 \mathrm{mM}$ 2-Deoxy glucose (DG) was added and the cells incubated for $20 \mathrm{~min}$. The amount of glucose uptake was determined as per manufactures protocol using the Glucose uptake kit from Biovision (glucose uptake colorimetric assay kit, the 2-DG6P is oxidized to generate $\mathrm{NADPH}$, which can be determined by an enzymatic recycling amplification reaction, color generated can be quantified colorimetrically at $412 \mathrm{~nm}$.). The calculation was carried out keeping 100\% glucose uptake for Pioglitazones (PGZ) was used as a standard drug.

$\checkmark \quad$ 2-DG uptake $=\mathrm{Sa} / \mathrm{Sv}(\mathrm{pmol} / \mu \mathrm{l}$ or $\mathrm{nmol} / \mathrm{ml}$ or $\mu \mathrm{M}$ )

$\checkmark$ Where: $\mathrm{Sa}$ is the amount of 2-DG6P (in pmol) in sample well calculated from Standard Curve.

$\checkmark \mathrm{Sv}$ is sample volume (in $20 \mu \mathrm{l}$ ) added into the sample well. antidiabetic activity of the synthesized derivatives was performed by the Glucose uptake assay and the results were tabulated below. 
In-vitro Antidiabetic Activity:

Table 2: Effect of compounds (SRT) on 2-DG uptake in 3T3-L1 presence and absence of insulin:

\begin{tabular}{|c|c|c|c|c|}
\hline S.No & Compound & OD (412) & 2DG6P(pmol) & 2-DGuptak(Pmol/ $\mu \mathrm{l})$ \\
\hline 1 & Insulin (1 micro Mol) & 3.04 & 174 & 11.5 \\
\hline 2 & SRT 1 & 4.5 & 70 & 5.0 \\
\hline 3 & SR + Insulin & 6.0 & 90 & 7.5 \\
\hline 4 & SRT 2 & 5.5 & 80 & 6.0 \\
\hline 5 & SR + Insulin & 7.0 & 115 & 8.5 \\
\hline 6 & SRT 3 & 3.5 & 60 & 4.0 \\
\hline 7 & SR + Insulin & 5.0 & 80 & 6.5 \\
\hline 8 & SRT 4 & 2.5 & 40 & 3.0 \\
\hline 9 & SR + Insulin & 4.0 & 70 & 5.5 \\
\hline 10 & SRT 5 & 0.6 & 10 & 1.0 \\
\hline 11 & SR + Insulin & 1.5 & 30 & 2.5 \\
\hline 12 & SRT 6 & 1.0 & 15 & 1.5 \\
\hline 13 & SR+ Insulin & 2.6 & 50 & 3.5 \\
\hline 14 & SRT 7 & 4.0 & 65 & 4.5 \\
\hline 15 & $\mathrm{SR}+$ Insulin & 5.5 & 85 & 7.0 \\
\hline 16 & SRT 8 & 1.5 & 30 & 2.0 \\
\hline 17 & SR + Insulin & 3.0 & 60 & 4.0 \\
\hline 18 & SRT 9 & 3.0 & 50 & 3.5 \\
\hline 19 & SR + Insulin & 4.5 & 75 & 6.0 \\
\hline 20 & SRT 10 & 5.0 & 75 & 5.5 \\
\hline 21 & $\mathrm{SR}+$ Insulin & 6.5 & 110 & 8.0 \\
\hline 22 & SRT 11 & 2.0 & 35 & 2.5 \\
\hline 23 & SR + Insulin & 3.5 & 65 & 4.5 \\
\hline 24 & SRT 12 & 6.0 & 90 & 6.5 \\
\hline 23 & $\mathrm{SR}+$ Insulin & 7.0 & 120 & 9.0 \\
\hline 10 & $\begin{array}{l}\text { Pioglitazone(10Micro.Mol) } \\
\text { +Insulin (1 Micro.Mol) }\end{array}$ & 10.4 & 450 & 22.5 \\
\hline 11 & $\begin{array}{l}\text { Pioglitazone } \\
\text { (10 Micro.Mol) }\end{array}$ & 3.1 & 210 & 10.5 \\
\hline
\end{tabular}

\section{Summary and Conclusion:}

In the present research studies, based on the huge literature survey, we designed novel derivatives and screened for their insilico and Invitro methods. We performed Docking for Anti diabetric activities by comparing with
Standard Drugs. All the results obtained by insilico and Invitro are in satisfactory manner, containing very near to each other. Based on this promising in-vitro anti-diabetic results, also give scope for further studies. Further research need to be carried out to know the relationship between structure and biological activity 


\section{SCHEME}<smiles>C[14C](C)(Nc1nc2ccc(F)c(Cl)c2s1)C(=O)Nc1ccc(S(C)(=O)=O)cc1</smiles>

$$
\begin{aligned}
\mathrm{R} & =0, \mathrm{~m}, \mathrm{p}-\text { chloro aniline } \\
& =0, \mathrm{~m}, \mathrm{p}-\text { nitro aniline } \\
& =\text { aniline } \\
& =\mathrm{PABA} \\
& =\text { Morpholine, piperazine } \\
& =\text { Dimethylanine, Diphenyl amine }
\end{aligned}
$$

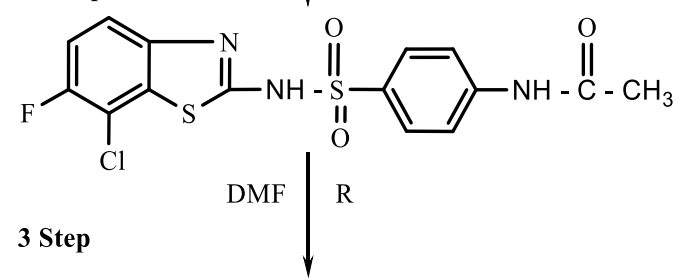
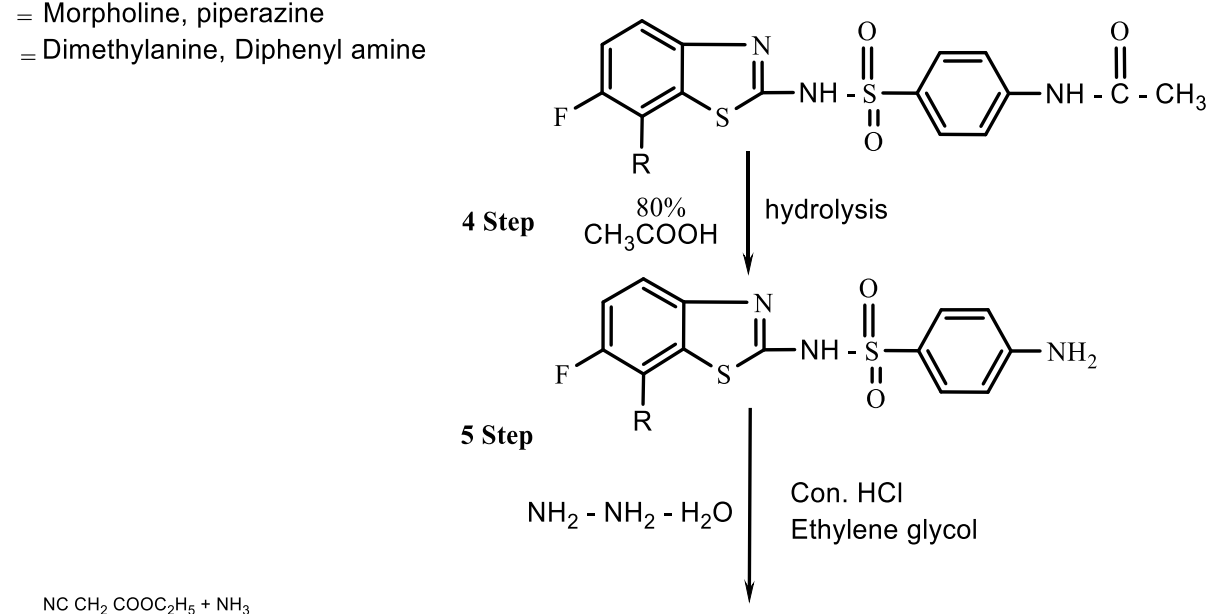

$$
\begin{aligned}
& \mathrm{NC} \mathrm{CH} \mathrm{COOC}_{2} \mathrm{H}_{5}+\mathrm{NH}_{3}
\end{aligned}
$$

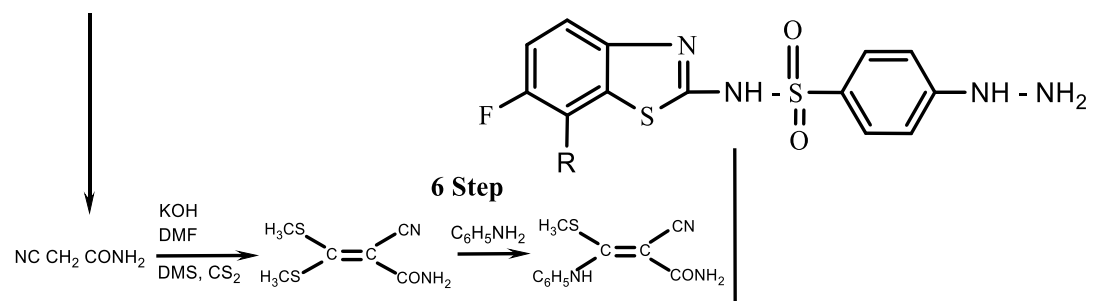

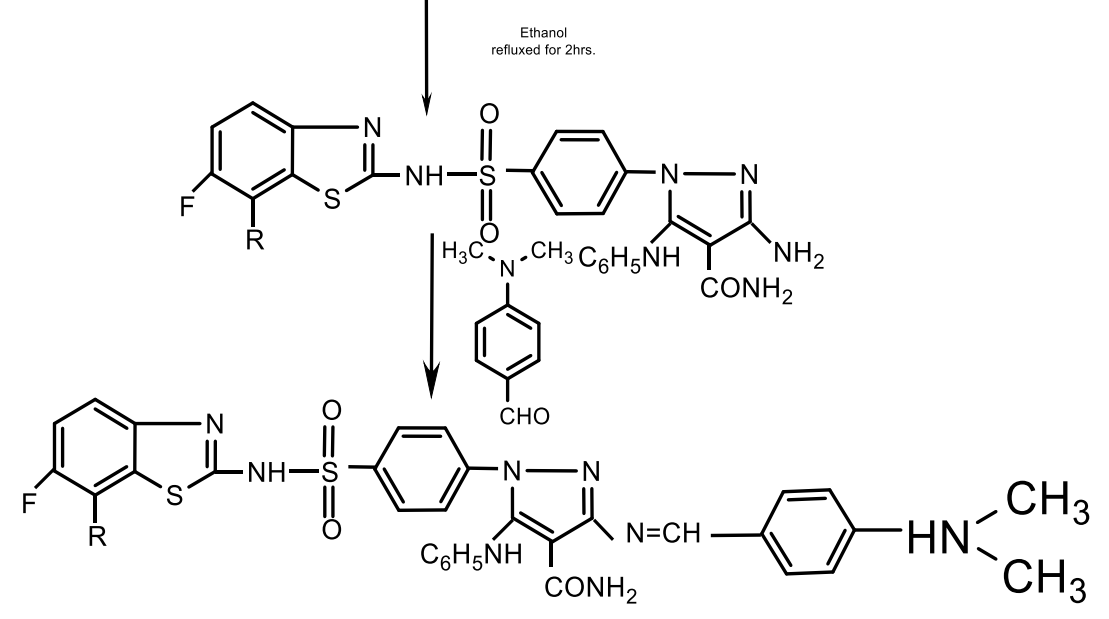




\section{References:}

1. Fluorine in Organic Chemistry Richard D. Chambers 2004 Blackwell Publishing Ltd. ISBN: 978-1-405-10787-7.

2. Naira Nayeem*, Gladsy Denny Synthesis of some Mannich bases from sulphonamides and benzothiazole derivatives and evaluation of their anti tubercular activity and anti microbial activity Der Pharma Chemica, 2012, 4 (3):1277-1282

3. Reena mahtab, Ashish srivastava, Nishi gupta, Sandeep kumar kushwaha, Aradhna tripathi Synthesis of novel 2-benzylbenzo[d] thiazole6-sulfonamide derivatives as potential anti inflammatory agent Journal of Chemical and Pharmaceutical Sciences January 1(7) 2014.

4. Asif Husain, Diwakar Madhesia, Mohd Rashid, Aftab Ahmad \& Shah Alam Khan Synthesis and in vivo diuretic activity of some new benzothiazole sulfonamides containing quinoxaline ring system $\mathrm{J}$ Enzyme Inhib Med Chem, 2016; 31(6): 1682-1689

5. Iro Argyropoulou, Athina Geronikaki, Paola Vicini, and Franca Zani Synthesis and biological evaluation of sulfonamide thiazole and benzothiazole derivatives as antimicrobial agents ARKIVOC 2009 (vi) 89-102

6. Mathew George, Lincy Joseph, Ansa Thomas "Synthesis, characterization and biological screening of novel pyrazolone derivatives for certain pharmacological activities" International Journal of Pharmaceutical Science and Research, Volume 2; Issue 4; July 2017.

7. Honnalagere ramesh puneeth, Angatahally chandrashekariah sharada "Antidiabetic effects of a series of curcumin pyrazoles invitro" Asian Journal of Pharmaceutical and Clinical Research, Vol 8, Issue 6,146-149, 2015

8. PrasannaA.DatarandSonaliR.Jadhav "Design and Synthesis of Pyrazole-3-one Derivatives as Hypoglycaemic Agents" International Journal of Medicinal Chemistry, Article ID 670181,2015 .
9. Mohd. Javed Naim, Ozair Alam, Md. Jahangir Alam, Mohammad Shaquiquzzaman, Md. Mumtaz Alam, Vegi Ganga Modi Naidu "Synthesis, docking, in vitro and in vivo antidiabetic activity of pyrazole-based 2,4thiazolidinedione derivatives as PPAR- $\gamma$ modulators" Arch Pharm Chem Life Sci.e1700223,2018.

10. Hassan M. Faidallah, Manal M. A1Mohammadi, Khalid A. Alamry \& Khalid A. Khan "Synthesis and biological evaluation of fluoropyrazolesulfonylurea and thiourea derivatives as possible antidiabetic agents" Journal of Enzyme Inhibition and Medicinal Chemistry,31(S1): 157-163,2016

11. Book on Molecular docking and molecular dynamic techniques to achieve rational drug design by Amalia stefaniu, intechopen 2019.

12. Ayaz Mahmood Dar and Shafia Mir Molecular Docking: Approaches, Types, Applications and Basic ChallengesJournal of Analytical \& Bioanalytical Techniques 2017, 8:2

13. Xuan-Yu Meng, Hong-Xing Zhang, Mihaly Mezei, and Meng Cui Molecular Docking: A powerful approach for structure-based drug discovery Curr Comput Aided Drug Des . 2011 June 1; 7(2): 146-157

14. Gerhard Vogel, Drug discovery and evaluation: Pharmacological assays, p:15711577.

15. Yamamoto, N. et al. (2015) Measurement of glucose uptake in cultured cells. Current Protocols in Pharmacology. 71, 12.14.1-26

16. Michael P Valley, Natasha Karassina, Natsuyo Aoyama, Coby Carlson, James J Cali, Jolanta Vidugiriene A bioluminescent assay for measuring glucose uptake Analytical Biochemistry 505, 43-50

17. Kumiko Saito, Suni Lee, Tetsuya Shiuchi, Chitoku Toda, Masahiro Kamijo, Kyoko Inagaki-Ohara, Shiki Okamoto, Yasuhiko Minokoshi An enzymatic photometric assay for 2-deoxyglucose uptake in insulin-responsive tissues and 3T3-L1 adipocytes Analytical Biochemistry 412, 9-17 